\title{
Changes in the Distribution of
} Intrauterine Microbiota May Attribute to Immune Imbalance in the CBA/J xDBA/2 Abortion-Prone Mice Model



OPEN ACCESS

Edited by:

Sha Wu,

Southern Medical University, China

Reviewed by:

Rong Zhou,

Sichuan University, China

Chong Qiao,

Shengjing Hospital of China Medical

University, China

*Correspondence:

Jianping Tan

julietp2004@126.com

Jianping Zhang

zhjianp@mail.sysu.edu.cn

Hui Chen

chenhui9@mail.sysu.edu.cn

tThese authors have contributed equally to this work

Specialty section:

This article was submitted to

Immunological Tolerance and

Regulation,

a section of the journal

Frontiers in Immunology

Received: 14 December 2020

Accepted: 16 February 2021

Published: 08 March 2021

Citation:

Bai S, Huang B, Fu S, Zhu M, Hu L,

Zhu L, Chen M, Zhang Z, Tan J,

Zhang $J$ and Chen $H$ (2021) Changes

in the Distribution of Intrauterine

Microbiota May Attribute to Immune

Imbalance in the $C B A / J \times D B A / 2$

Abortion-Prone Mice Model.

Front. Immunol. 12:641281.

doi: 10.3389/fimmu.2021.641281

\begin{abstract}
'Department of Obstetrics and Gynecology, Sun Yat-sen Memorial Hospital, Sun Yat-sen University, Guangzhou, China, ${ }^{2}$ Center for Reproductive Genetics and Reproductive Medicine, Sun Yat-sen Memorial Hospital, Sun Yat-sen University, Guangzhou, China, ${ }^{3}$ Department of Radiation Oncology, Shenzhen Traditional Chinese Medicine Hospital, The Fourth Clinical Medical College of Guangzhou University of Chinese Medicine, Shenzhen, China
\end{abstract}

Background: Female Genital Tract (FGT) is an important micro-ecological area of human body. Microbiota in the lower reproductive tract may subsequently invade the uterine cavity during embryo implantation and produce immune responses. CBA J ×DBA/2 mating combination has been widely used as an abortion-prone mice model but whether microbiota existed in their uterine cavity remains unclear. In this context, the role of the microbial communities in immune response deserves attention.

Objective: To investigate the relationship between the distribution of microbiota in the uterine cavity of $\mathrm{CBA} / \mathrm{J} \times \mathrm{DBA} / 2$ abortion-prone mouse model and the immune imbalance of the maternal-fetal interface.

Methods: In this study, female CBA $/ J$ mice were paired with male DBA/2 mice to develop an abortion-prone model (BA group), and with male BALB/c mice to build a standard pregnancy model (BC group). The non-pregnant female mice were served as the control group (C group). Uterine flushing fluid and sera were collected on day 13.5 of pregnancy. 16S rRNA sequencing technology was used to analyze the distribution of intrauterine microbiota. Phylogenetic Investigation of Communities were conducted to predict the microbiota functions by Reconstruction of Unobserved States (PICRUST) and Kyoto Encyclopedia of Genes and Genomes (KEGG). The serum IL 10, INF- $\gamma$, and TNF- $\alpha$ levels were examined using Enzyme-linked immunosorbent assay (ELISA) method.

Results: All samples were detected with microbial communities. The $\alpha$ diversity $p$ $=0.00077$ ) had significant differences among three groups. Proteobacteria was the most dominant phylum in C group (mean $=83.21 \%$ ) and BA group ( mean $=43.23 \%$ ). Firmicutes was dominant in BC group (mean $=46.4 \%$ ), as well as the second dominant one in $\mathrm{C}$ group (mean $=12.63 \%$ ) and BA group (mean $=40.55 \%$ ). Microbiota functions were associated with metabolism and immune response through the NOD-like receptor signaling pathway. The serum IL 10 level in BA group were significantly lower than that in BC group (10.14 $\pm 1.90 \mathrm{pg} / \mathrm{ml}, n=8$; vs. $19.03 \pm 1.82 \mathrm{pg} / \mathrm{ml}, n=10 ; p=0.004$ ). 
The serum TNF- $\alpha$ and INF- $\gamma$ level in BA group were also significantly higher than that in $\mathrm{BC}$ group $(523.1 \pm 58.14 \mathrm{pg} / \mathrm{ml}, n=8$ vs. $310.3 \pm 28.51 \mathrm{pg} / \mathrm{ml}, n=10, p=0.0029$ $69.22 \pm 5.38 \mathrm{pg} / \mathrm{ml}, n=8$ vs. $50.85 \pm 2.45 \mathrm{pg} / \mathrm{ml}, n=10, p=0.0042)$.

Conclusion: Microbial communities were colonized in uterine cavity of $\mathrm{CBA} / \mathrm{J}$ mice both at non-pregnant stage and pregnant stage when mated with both BALB/C and DBA/2 male mice. The differentially abundant microbiome may be attributed to the immune tolerance through binding to the NOD-like receptor.

Keywords: microbiota, 16S rRNA sequencing, abortion-prone model, immune imbalance, maternal-fetal interface

\section{INTRODUCTION}

Maintaining pregnancy requires an immune balance microenvironment at the maternal-fetal interface. Innate immunity and acquired immunity jointly contribute to this immune tolerance microenvironment, while an imbalanced stage could lead to pregnancy loss (1). The surviving embryos are prone to placental related diseases like intrauterine fetal growth restriction and hypertension during pregnancy (2). Many factors may break the immune balance and microbial invasion that could activate the innate immunity. The Female Genital Tract (FGT) is an important micro-ecological area in human body, hosting many microorganisms and closely linked to human health. Traditionally, uterine cavity has been often known as a classic sterile cavity. However, Recent studies have found a certain amount of symbiotic microbiota in the uterine cavity and some of them only found in the uterine cavity (3). Besides, microbiota from the lower reproductive tract can enter the uterine cavity and stimulate trophoblasts to produce an immune response at the stage of embryo implantation.

NLR receptor (NOD-like receptor) family, as a major cytoplasmic PPR (Pattern recognition receptors), plays an important role in the innate immune system, maintaining the intracellular environment. It plays a defensive function by identifying PAMPs (pathogen-associated molecular pattern) and endogenous DAMPs (damage-associated molecular patterns) that invade cells (4). Recent studies have shown that the degradation products of bacterial cell wall peptidoglycan (PGN) can be recognized by both NOD1 and NOD2 proteins, and the PGN motifs they recognize are distinct. The smallest structure recognized by NOD1 is $\gamma$-D-glutamic acid-mesodiaminopimelic acid ( $\gamma$-D-glu-meso-DAP, mesoDAP), which mainly exists in the PGN of $\mathrm{G}^{-}$bacteria. Therefore, NOD1 can specifically recognizes $\mathrm{G}^{-}$bacteria. The smallest structure recognized by NOD2 is MDP (muramyl dipeptide) which exists in the PGN of all bacteria, thus NOD2 is both a receptor for $\mathrm{G}^{+}$bacteria and $\mathrm{G}^{-}$bacteria (5). Our previous study confirmed that URSA (Unexplained Recurrent Spontaneous Abortion) patients have high expressions of NOD1 and NOD2, affecting the behavior of trophoblasts through the MAPK/p38 signaling pathway (6). The CBA/J'DBA/2 mice model has been commonly employed to investigate immune tolerance at the maternal-fetal interface with pregnancy failure. Here we aim to explore whether there is a linkage between the distribution of intrauterine microbiota and immune imbalance at the maternal-fetal interface in the CBA/J'DBA/2 abortion mice model.

\section{MATERIALS AND METHODS}

\section{Animals and Treatment}

Ten to 12 weeks old of male DBA/2 mice, male BALB/c mice and female $\mathrm{CBA} / \mathrm{J}$ mice were purchased from Beijing HFK Bioscience Co., LTD. All animals were feed at the Center for Disease Model Animals of Sun Yat-sen University and maintained under a 12$\mathrm{h}$ light-dark cycle at constant temperature $\left(20-22^{\circ} \mathrm{C}\right)$ with free access to standard food and water. Female CBA/J mice were randomly allocated into abortion model group (BA group, $n=$ 8 ), normal pregnant control group (BC group, $n=10$ ), and normal non-pregnant control group (C group, $n=8$ ). Female mice and male mice were mated at 18:00 p.m. to construct a normal pregnancy model $(\mathrm{CBA} / \mathrm{J} \times \mathrm{BALB} / \mathrm{c})$ and an abortion model $(\mathrm{CBA} / \mathrm{J} \times \mathrm{DBA} / 2)$. Female $\mathrm{CBA} / \mathrm{J}$ mice detected vaginal plugs on day 0.5 of gestation. On day 13.5 of pregnancy, animals were killed by $\mathrm{CO}_{2}$ inhalation. Embryo absorption rate were measured in order to determine the accuracy of the abortion model. All animal experimental protocols were approved by the Animal Ethics Committee of Sun Yat-Sen University (Guangzhou, China).

\section{Sample Collection}

The uterus was separated from the cervix and fallopian tubes aseptically. One ml RNase- and DNase-free water was injected from one side of uterine horn and flushing fluid was collected in cryopreservation tube, placed into liquid nitrogen, and then transferred to $-80^{\circ} \mathrm{C}$ for future analysis (Figure 1). Samples were collected under sterile conditions to avoid contamination. Puncturing into the amniotic cavity had avoided any interference with the amniotic fluid. Due to the difficulty of obtaining materials for uterine flushing fluid, we only collected 2 cases in $\mathrm{C}$ group, 5 cases in $\mathrm{BA}$ group and 5 cases in $\mathrm{BC}$ group.

\section{DNA Extraction and 16S rRNA Sequencing}

Genomic DNA were extracted from the frozen uterine cavity fluid sample by QIAmp DNA mini-kit (Qiagen, USA) following the manufacturer's protocol. Sterile saline was chosen as the negative control. The V3-V4 region of the $16 \mathrm{~S}$ rRNA gene sequencing was completed by MAGIGENE (Guangzhou, China) 


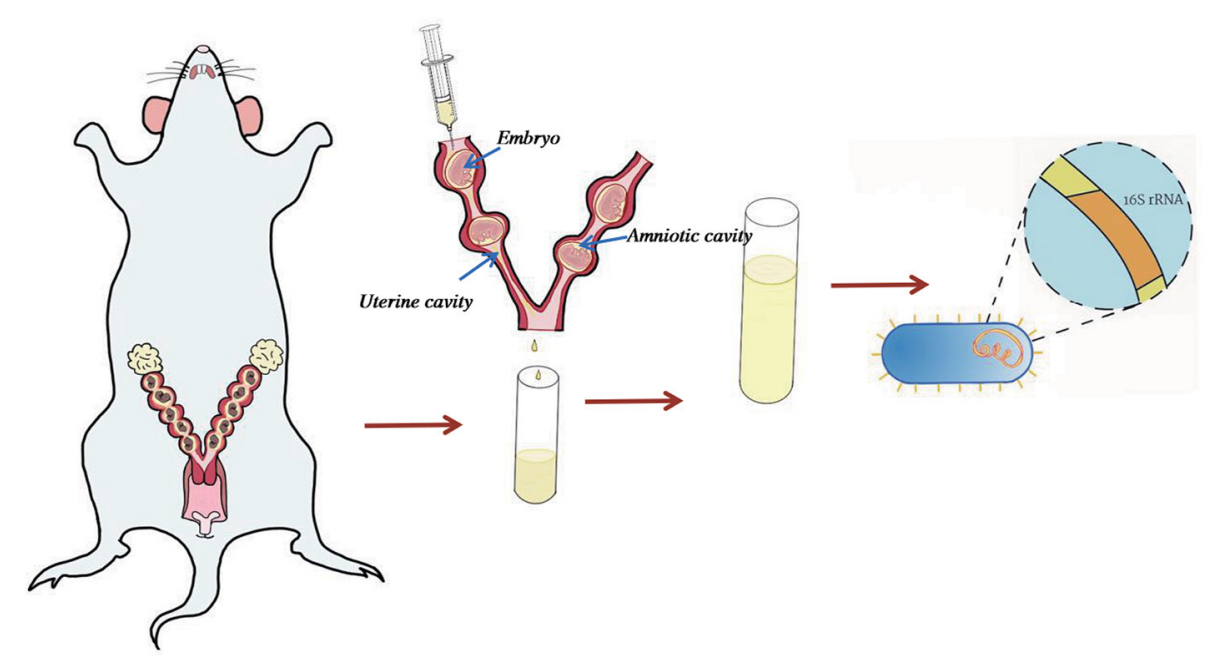

FIGURE 1 | Sample collection method. Uterus was separated from the cervix and fallopian tubes. $1 \mathrm{ml}$ RNase- and DNase-free water was used to rinse the uterine cavity to avoid piercing the amniotic cavity or piercing the uterine wall. The flushing fluid sample was collected for 16 s rRNA sequence.

according to the standard service workflow. Primers 357-F (5'-ACTCCTACGGRAGGCAGCAG-3') and 806R (5' -GGACTA CHVGGGTWTCTAAT-3') was used to amplify $16 \mathrm{~S}$ rRNA gene by PCR following the Illumina miseq protocols. PCRs were run in duplicate using GeneAmp ${ }^{\circledR}$ PCR System 9700 (California, USA) using thermocycling conditions of $94^{\circ} \mathrm{C}$ for $2 \mathrm{~min}, 38$ cycles of $94^{\circ} \mathrm{C}$ for $30 \mathrm{~s}, 56^{\circ} \mathrm{C}$ for $30 \mathrm{~s}, 72^{\circ} \mathrm{C}$ for $30 \mathrm{~s}$, a final extension at $72^{\circ} \mathrm{C}$ for $5 \mathrm{~min}$, and a final temperature at $10^{\circ} \mathrm{C}$. PCR products were checked by agarose gel electrophoresis and collected using AxyPrepDNA (Huangzhou, China). The PCR products were quantified using FTC-3000 TM real-time PCR and sequenced using MiSeq ${ }^{\circledR}$ Reagent Kit v3 (Illumina) on a MiSeq-Illumina platform (Lifesequencing sequencing service, Valencia, Spain).

\section{Bioinformatics and Data Analysis}

Sequences were processed through QIIME (7). Based on their sequence similarity using UPARSE, all sequences were clustered into operational taxonomic units (OTU), with a threshold of sequence similarity as 0.97 . Singletons and OTUs with a relative frequency below 0.01 were removed. Alpha diversity was evaludated using QIIME and Shannon method was applied to analyze the biodiversity of samples. The $\mathrm{X}$-axis represented the grouping, and the Y-axis represented the corresponding Shannon diversity index. The shape on the plots show the richness of alpha diversity that the higher Y-axis values suggested the richer species (Figure 3A).

Beta diversity was conducted using Principal coordinates analysis (PCoA) plots. The LEfSe method (8) was applied to analyze and identify the differentially abundant microbiome across three groups. Linear discriminant analysis (LDA) was employed to identify communities or species with significant differences in the division of samples into different groups, depending on the taxonomic composition. The total statistical analysis on bacterial taxonomic identification was performed using Calypso software (version 8.10).

\section{PICRUST and KEGG Enrichment}

Functional Potential of the Microbiome was used PICRUST and the GO and KEGG database were adopted by putting in the OTU abundances (9).

\section{Enzyme-Linked Immunosorbent Assay (ELISA)}

Sera were collected after centrifugation of blood obtained from the abdominal aortic puncture and stored at $-80^{\circ} \mathrm{C}$. The serum IL 10, INF- $\gamma$ and TNF- $\alpha$ levels were examined using ELISA method following manufacturer's instruction.

\section{Data Analysis}

Statistical analysis was performed using SPSS 19.0. Measurement data were expressed as mean \pm standard deviation $(X \pm s)$. The mean comparisons of serum IL 10, INF- $\gamma$ and TNF- $\alpha$ levels between two groups were performed by $t$-test. The comparison of the rate of two groups was performed using Pearson Chisquare test. Alpha diversity was analyzed using the analysis of variance (ANOVA) method. $P<0.05$ was considered to be statistically significant.

\section{RESULTS}

\section{Abortion-Prone Model and Embryo Resorption Rate}

The embryo resorption rate of BA group was $27.51 \pm 3.652 \%$, which was significantly higher than that in BC group $(2.74 \pm$ $1.68 \%, P<0.0001$ ) (Figure 2A). Following our previous study, the current abortion mice model was considered successful.

\section{The Level of Inflammatory Factors in the Serum}

The serum IL-10 level in BA group was significantly lower than that in $\mathrm{BC}$ group $(10.14 \pm 1.90 \mathrm{pg} / \mathrm{ml}, n=8$; vs. $19.03 \pm 1.82$ 
A
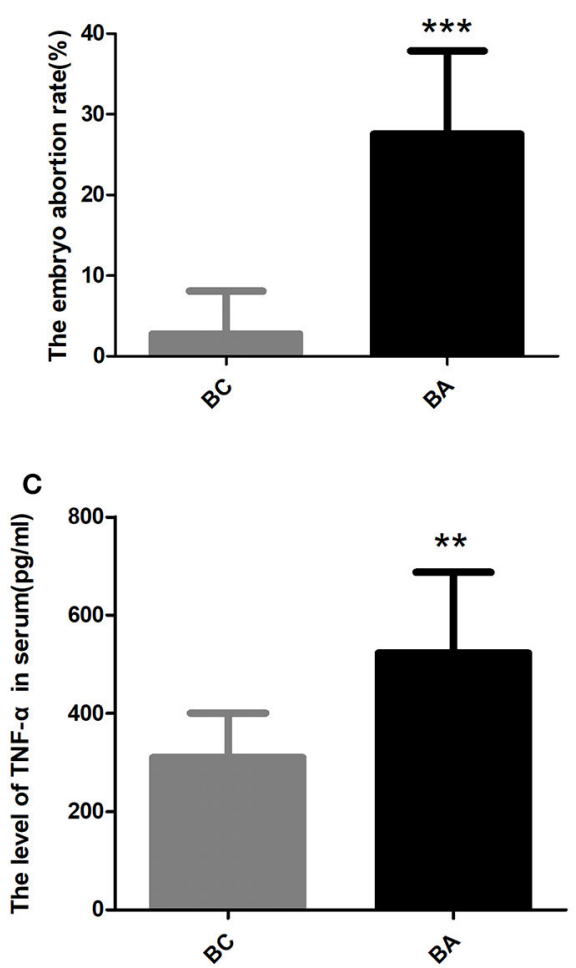

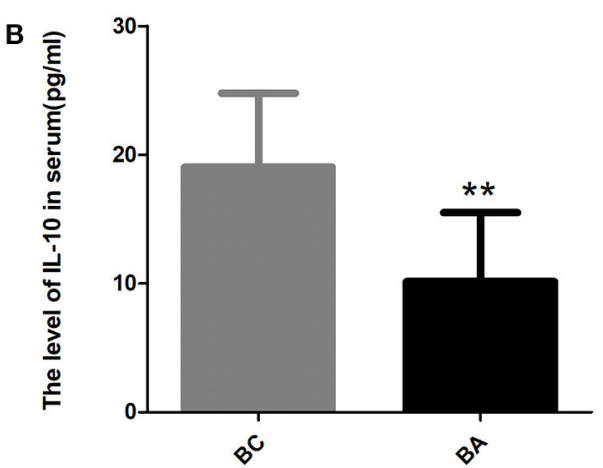

D

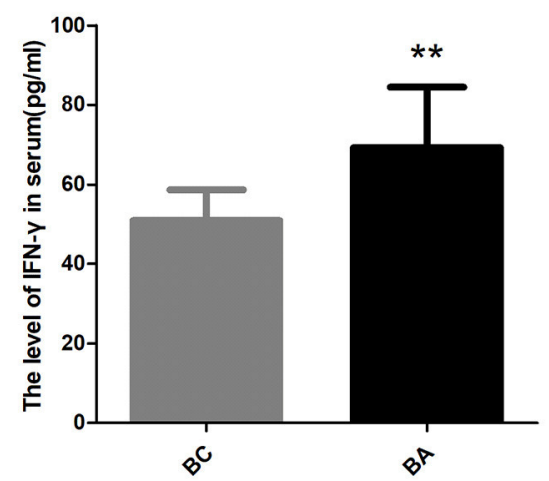

FIGURE 2 | The embryo abortion rate and the level of inflammatory factors in the serum. (A) The embryo abortion rate in BC group was significantly lower than that in BA group (2.74 $\pm 1.68 \%$ vs. $27.51 \pm 3.652 \%, P<0.0001)$. (B) The serum IL-10 level in BA group were significantly lower than that in BC group (10.14 $\pm 1.90 \mathrm{pg} / \mathrm{ml}$, $n=8$; vs. $19.03 \pm 1.82 \mathrm{pg} / \mathrm{ml}, n=10 ; p=0.004)$. (C) the serum TNF- $\alpha$ level in BA group (523.1 $\pm 58.14 \mathrm{pg} / \mathrm{ml}$ ) were significantly higher than that in $\mathrm{BC}$ group $(310.3 \pm 28.51 \mathrm{pg} / \mathrm{ml}, p=0.0029)$. (D) The serum INF- $\gamma$ level in BA group (69.22 $\pm 5.38 \mathrm{pg} / \mathrm{ml})$ were significantly higher than that in $\mathrm{BC}$ group $(50.85 \pm 2.45 \mathrm{pg} / \mathrm{ml}$, $p=0.0042){ }^{* *}$ indicates $P<0.01 ;{ }^{* * *}$ indicates $P<0.001$.

$\mathrm{pg} / \mathrm{ml}, n=10 ; p=0.004$ ) (Figure 2B). However, the serum TNF$\alpha$ level in BA group $(523.1 \pm 58.14 \mathrm{pg} / \mathrm{ml})$ were substantially higher than that in BC group $(310.3 \pm 28.51 \mathrm{pg} / \mathrm{ml}, p=0.0029)$ (Figure 2C). The serum INF- $\gamma$ level between two groups had the same trend, with the level in BA group $(69.22 \pm 5.38 \mathrm{pg} / \mathrm{ml})$ significantly higher than that in BC group $(50.85 \pm 2.45 \mathrm{pg} / \mathrm{ml}$, $p=0.0042$ ) (Figure 2D).

\section{Dataset Features and Bacterial Diversity}

A total of 391,417 of $16 \mathrm{~S}$ rRNA effective raw reads were generated from the 11 samples, and the clean raw reads optimized after were 138,352, Supplementary Table 1). The sequence depth per group varied, with the highest number of clean raw reads obtained from BA group (mean $=14881.4$ ) and BC group (mean $=14965.25)$. C group obtained the least clean raw reads $($ mean $=2,042)$ (Supplementary Table $\mathbf{1})$. These reads were assigned to 127 OTUs. Alpha-diversity was calculated by Shannon index according to obtained reads and OTUs as shown in Figure 3A. The BA group had the richest bacterial diversity, followed by the $\mathrm{BC}$ group. The $\mathrm{C}$ group has the smallest Shannon index, suggesting a comparatively simple microbial community in this group. Significant differences in the alphadiversity were identified across the three groups $(p=0.00077)$. Principal coordinate analysis indicated that samples within the same group were much more likely located closely on the PCoA plots (Figure 3B). Specifically, microbial communities in the uterine cavity of CBA/J mice were distinct before and during birth. Compared with the non-pregnant stage, pregnant CBA/J mice had more abundant microbial populations, and the degree of diversity was more significant when combined with DBA/2 mice. The intra-group differences in the three groups of samples were small.

\section{Characteristics of Intrauterine Microbiome in CBA/J Mice}

Proteobacteria was the most dominant phylum in $\mathrm{C}$ group $($ mean $=83.21 \%)$ and BA group $($ mean $=43.23 \%)($ Figure $3 C)$. Firmicutes was dominant in BC group (mean $=46.4 \%$ ) and the second dominant in both $\mathrm{C}$ group (mean $=12.63 \%$ ) and BA group $($ mean $=40.55 \%)$. Figure 3D showed a heat map of relative abundances of OTUs at the genus level. Ralstonia was the most abundant in C group (mean $=42.21 \%$ ). Phyllobacterium, Staphylococcus as well as Vibrio and Pseudomonas were typical 
A

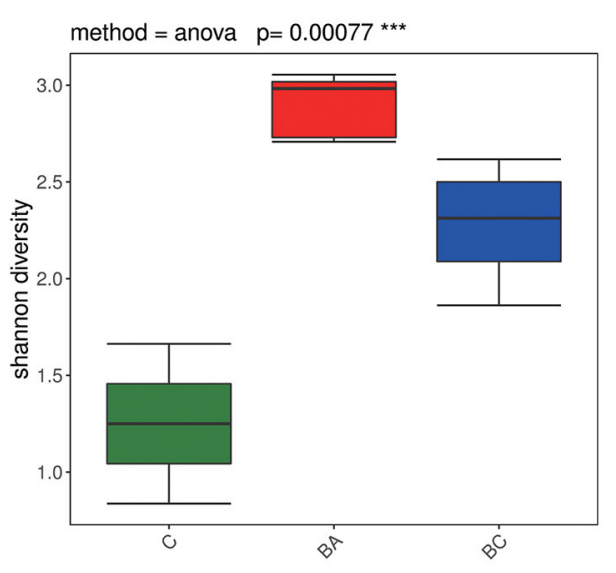

C

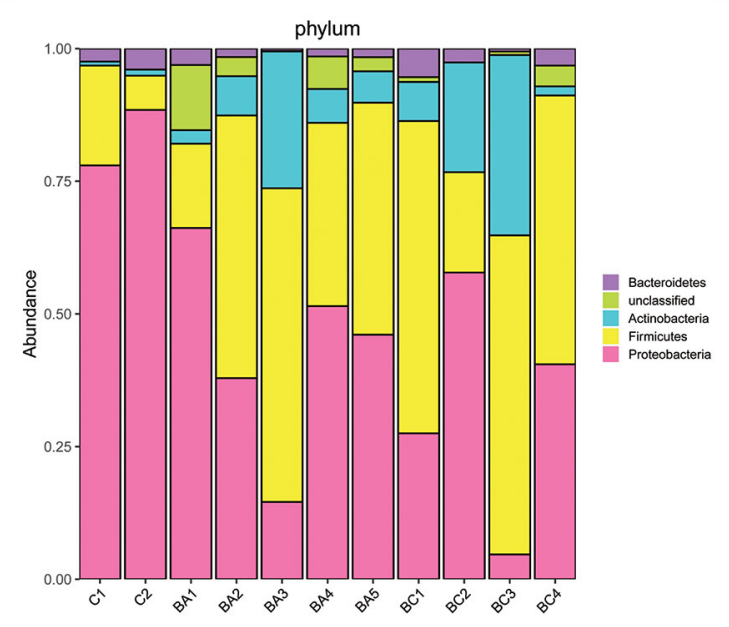

B

D
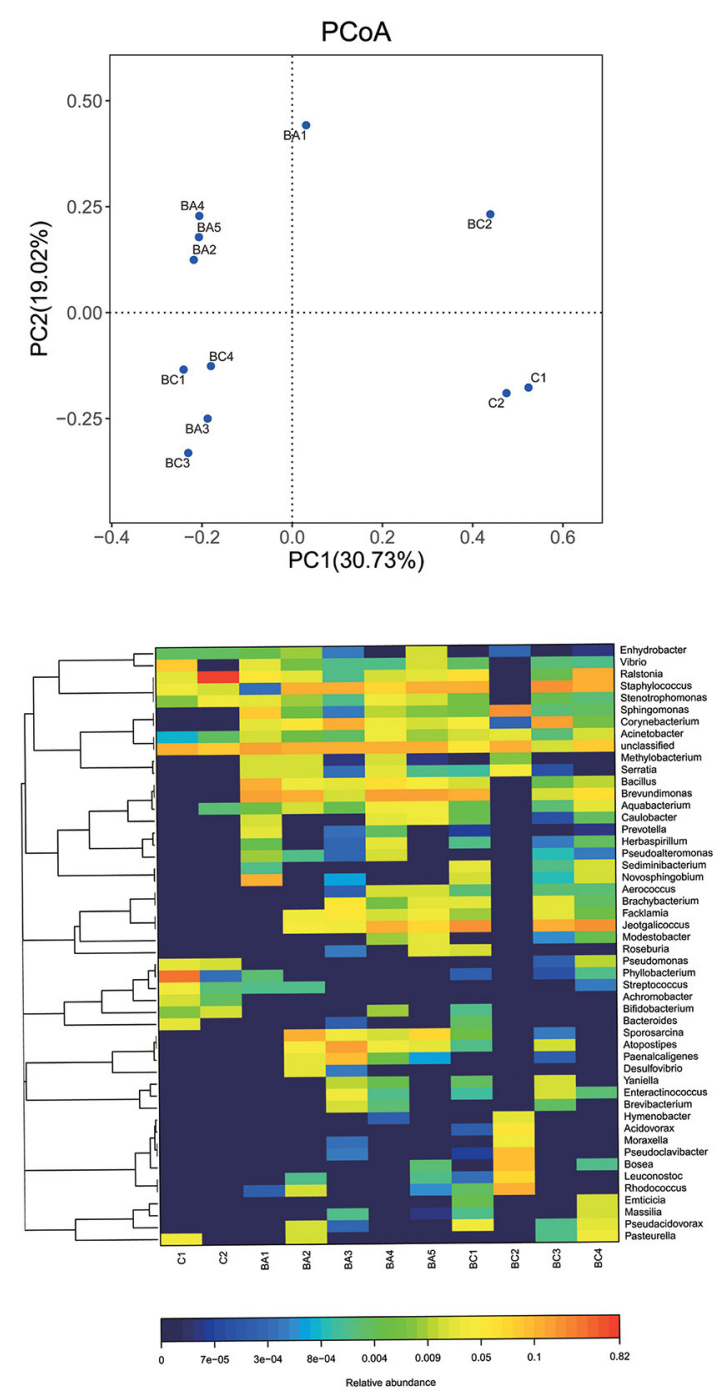

FIGURE 3 | Bacterial composition and diversity. (A) Alpha diversity was significantly different across three groups $(P<0.001)$. The C group had the least species of microbiome and the BA group had the largest. (B) Samples of each group were located closely on the PCoA plots. (C) The composition of microbial communities at phylum level. (D) The composition of microbial communities at genus level.

taxa. Acinetobacter, Aquabacterium, Sphingomonas, Pasteurella, and Achromobacter were also observed but at relatively low abundances of the total population. BA group had an abundance of Brevundimonas (mean $=16.5 \%$ ) followed by Staphylococcus, Corynebacterium, Bacillus, and Jeotgalicoccus. In BC group, the most dominant genus was Jeotgalicoccus (mean $=21.05 \%$ ) and the second dominant one was Staphylococcus (mean $=17.51 \%$ ). Corynebacterium, Sphingomonas, Ralstonia, and Brevundimonas were also found at lower relative abundances.

We then used LEfSe to classify various abundant microbiomes among three classes and found that Achromobacter, Pseudomonas and Bacteroidales were associated with discriminatory features in the $\mathrm{C}$ group, and Methylobacterium, Bacillus, Brevundimonas and Caulobacteraceae were associated with discriminatory features in group BA (Figures 4A,B). Comparing with $\mathrm{BA}$ group, $\mathrm{C}$ and $\mathrm{BC}$ group were more likely to have lower abundance or absence of Carnobacteriaceae (Figure 4C), Bacillus (Figure 4D), and Caulobacterales (Figure 4E).

\section{Functions of Intrauterine Microbiome in CBA/J Mice}

The different abundance of microbiota was primarily enriched in metabolic pathways such as Peptidases, Pyruvate metabolism and TCA cycle, through the PICRUST and KEGG enrichment study (Figure 5A). Regarding the function and role of microbial communities in the immune system, there were no difference 


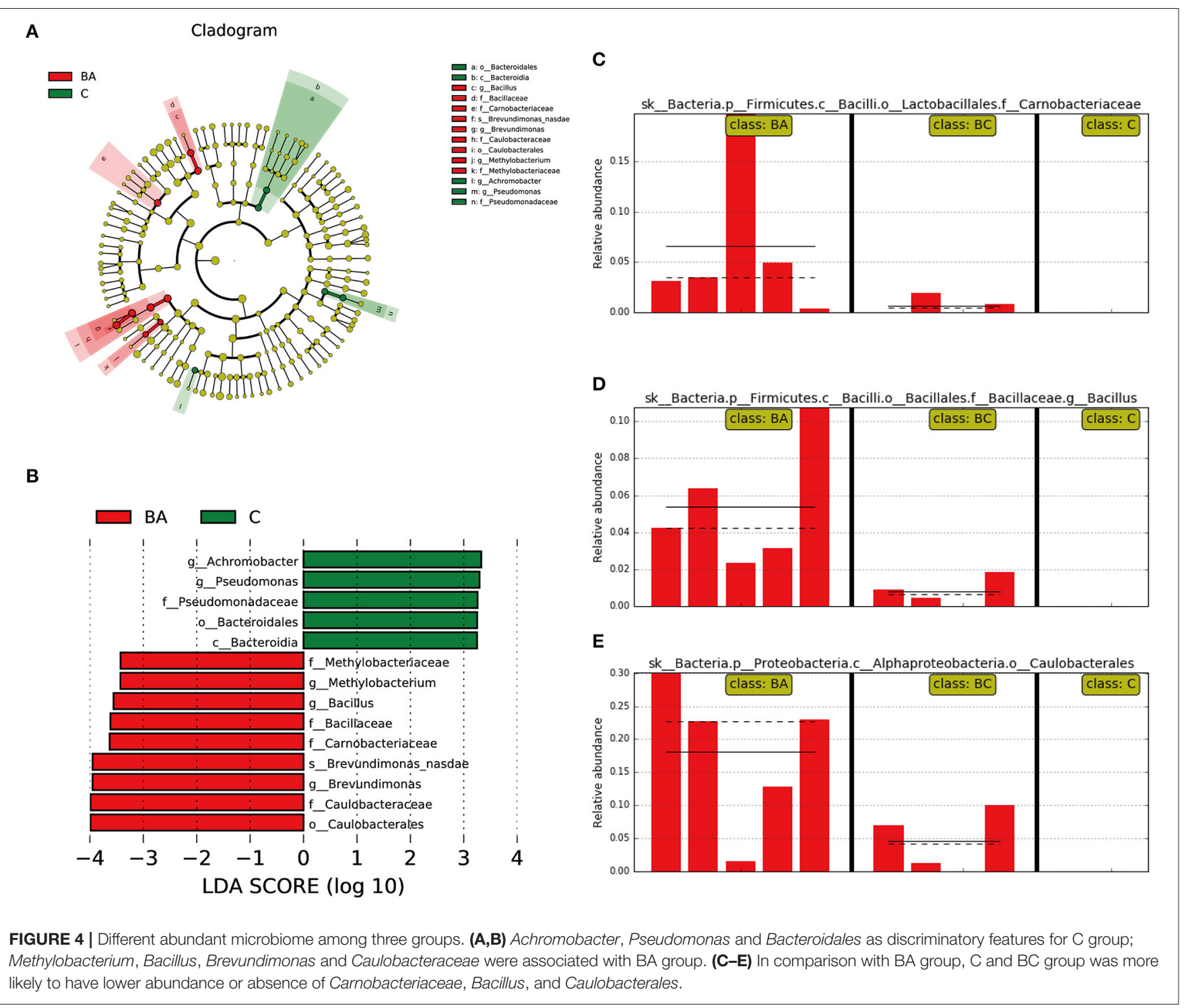

identified in NOD-like receptor signaling pathway across three groups ( $p=0.295$, Figure 5B).

\section{DISCUSSION}

To our knowledge, this is the first study to detect the microbiota in uterine cavity of CBA/J mice. Most research have focused on the composition of microbial communities in the human uterine cavity, amniotic fluid, placenta, and the downstream direction of causes of immune imbalance during pregnancy at the maternal-fetal interface. However, it is still necessary to clarify the composition, function, and relationship of microbial communities in the uterine cavity of $\mathrm{CBA} / \mathrm{J}$ mice as well as possible mechanisms of the associated immune imbalance. Here we utilize the 16S rRNA sequencing technology and PICRUST enrichment to provide additional insights on this topic. In particular, we found that the compositions of microbial communities in pregnant uterine cavity of $\mathrm{CBA} / \mathrm{J}$ mice were significantly distinct from those in unpregnant ones. When the mice were unpregnant, the diversity and the abundance of microbial communities were significantly lower. When the $\mathrm{CBA} / \mathrm{J}$ mice mated with different kinds of male mice, there were variations in the uterine microbial populations as well. Semen has to liquefy in the vagina before reaching the uterine cavity after passing through the cervix, so that the egg can be fertilized. In this process, the microbiota from the cervix and vagina, as well as those from the male mice may enter the uterine cavity and colonized (10). Our finding suggest that the uterine cavity microbiome may be affected by the lower reproductive tract microbial communities and the semen of male mice. Proteobacteria is reported to be one of the major bacterial genera significantly enriched in the endometrial cancer cohort (11). It is the dominant phylum in both samples taken from the vaginas and uteri from giant 


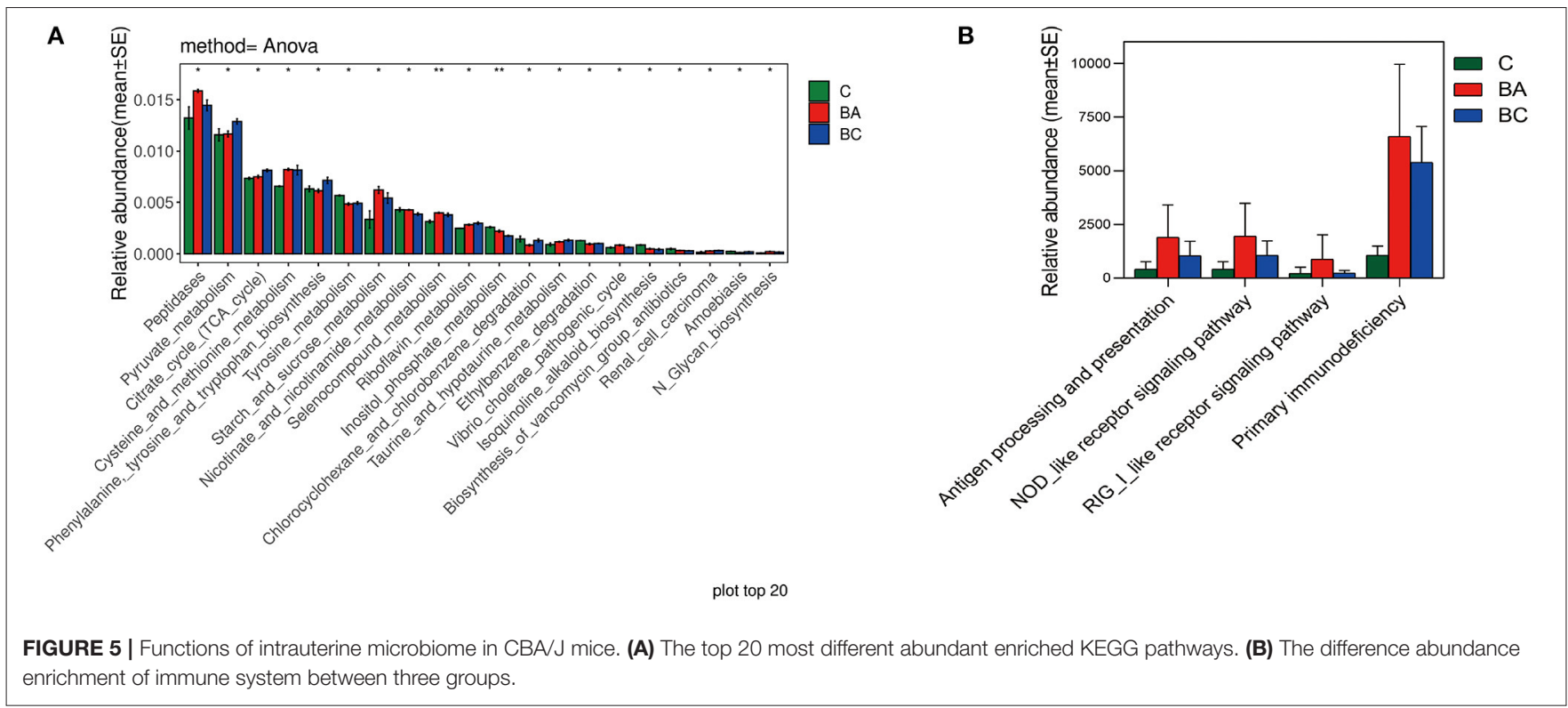

pandas and may be attributed to pregnancy outcomes (12). During pregnancy, fat and vitamin intakes could increase the abundance of Proteobacteria, which is a pro-inflammatory maternal microbiota (13). Tapiainen et al. showed that the most abundant phyla were Firmicutes followed by Proteobacteria in first-pass meconium of newborn infants, and they were affected by maternal factors during pregnancy implying that they were transferred from the uterus (14). In our study, we found that Proteobacteria was the most dominant phylum in both unpregnant $\mathrm{CBA} / \mathrm{J}$ mice and $\mathrm{CBA} / \mathrm{J} \times \mathrm{DBA} / 2$ abortionprone mice models. In $\mathrm{CBA} / \mathrm{J} \times \mathrm{BALB} / \mathrm{C}$ pregnant mice, the dominant phylum was Firmicutes. The abundance changes of Proteobacteria/Firmicutes may be a leading factor of abortionprone in mice model.

Furthermore, we compared the distribution of microbial communities across three groups. Interestingly, Carnobacteriaceae, Bacillus, and Caulobacterales were more abundant than the usual pregnant classes in the abortionprone model of mice whereas lacking in the unpregnant mice. Carnobacteriaceae may be attributed to hormone metabolites (15), which was increased in oral and gut samples after reinitiation of oral food intake after stroke (16). Bacillus was a spore-forming bacterium belonging to Firmicutes phylum, which can be activated in a suitable environment. Bacillus can be found in gastrointestinal tract of human and animals and stimulate the immune systems $(17,18)$. Caulobacterales belonging to Proteobacteria phylum can be obtained from soil and plants $(19,20)$. Future work should investigate whether they were the key bacteria leading to abortion and whether they can be used as biomarkers with potentially great clinical significance.

A normal pregnancy cannot be sustained without immune tolerance. The production of immune tolerance requires microbial colonization, therefore germ-free animals cannot achieve immune tolerance (21). However, when harmful microbial gains access to the uterine cavity, they can result in inflammation and disrupt the immune balance, eventually leading to abortion (22). We found the serum levels of IL-10, a representation of Th1 cytokines, were significantly lower in abortion-prone mice model whereas the serum level of TNF- $\alpha$ and INF- $\gamma$, a representation of Th2 cytokines, were significantly higher. This was consistent with previous studies (22-24). Regarding the role of microbial communities, we found that they can influence immune response through the NOD-like receptor signaling pathway, potentially because microbiome distribution changes could bind to the NOD-like receptor and participate in the Th1/Th2 immune balance bias that leads to abortion. Lastly, the following limitations should be considered. Samples were obtained from only uterine cavity, and we did not obtain the semen samples from different kinds of male mice, thus the composition of microbial communities among the three groups remain unclear. Besides, our data showed that microbial communities' functions were related to the signaling pathway of the NOD-like receptor, but the specific receptor remains unknown.

\section{CONCLUSION}

Our study explored the composition of microbial communities in uterine cavity of $\mathrm{CBA} / \mathrm{J}$ mice at both non-pregnant stage and pregnant stage, mated with $\mathrm{BALB} / \mathrm{c}$ male mice and DBA/2 male mice. The differentially abundant microbiome may be attributed to immune tolerance through binding to NOD-like receptor. The specific microbiota in the uterine cavity that could be characterized as the biomarker of abortion-prone bacteria and how it 
affects the immune response remains to be elucidated in future work.

\section{DATA AVAILABILITY STATEMENT}

The original contributions presented in the study are included in the article/Supplementary Material, further inquiries can be directed to the corresponding authors.

\section{ETHICS STATEMENT}

The animal study was reviewed and approved by All animal experimental protocols were approved by the Animal Ethics Committee of Sun Yat-Sen University (Guangzhou, China).

\section{AUTHOR CONTRIBUTIONS}

We declare that all the listed authors have participated actively in the study and all meet the requirements of the authorship. $\mathrm{HC}$ designed the study and wrote the protocol. SB and $\mathrm{BH}$ performed research. SF contributed important reagents. LZ and MC managed the literature searches and analyses. MZ and LH undertook the statistical analysis. JT, JZ, and ZZ wrote the first

\section{REFERENCES}

1. Trowsdale J, Betz AG. Mother's little helpers: mechanisms of maternal-fetal tolerance. Nat. Immunol. (2006) 7:241-6. doi: 10.1038/ ni1317

2. Renaud SJ, Postovit L-M, Macdonald-Goodfellow SK, McDonald GT, Caldwell JD, Graham CH. Activated macrophages inhibit human cytotrophoblast invasiveness in vitro. Biol. Reprod. (2005) 73:237-43. doi: 10.1095/biolreprod.104.038000

3. Moreno I, Codoner FM, Vilella F, Valbuena D, Martinez-Blanch JF, JimenezAlmazan J, et al. Evidence that the endometrial microbiota has an effect on implantation success or failure. Am. J. Obstet. Gynecol. (2016) 215:684-703. doi: 10.1016/j.ajog.2016.09.075

4. Mariathasan S, Weiss DS, Newton K, McBride J, O’Rourke K, Roose-Girma $\mathrm{M}$, et al. Cryopyrin activates the inflammasome in response to toxins and ATP. Nature. (2006) 440:228-32. doi: 10.1038/nature04515

5. Proell M, Riedl SJ, Fritz JH, Rojas AM, Schwarzenbacher R. The Nod-like receptor (NLR) family: a tale of similarities and differences. PLoS ONE. (2008) 3:e2119. doi: 10.1371/journal.pone.0002119

6. Wang Z, Liu M, Nie X, Zhang Y, Chen Y, Zhu L, et al. NOD1 and NOD2 control the invasiveness of trophoblast cells via the MAPK/p38 signaling pathway in human first-trimester pregnancy. Placenta. (2015) 36:652-60. doi: 10.1016/j.placenta.2015.03.004

7. Caporaso JG, Kuczynski J, Stombaugh J, Bittinger K, Bushman FD, Costello EK, et al. QIIME allows analysis of high-throughput community sequencing data. Nat. Methods. (2010) 7:335-6. doi: 10.1038/nmeth.f.303

8. Segata N, Izard J, Waldron L, Gevers D, Miropolsky L, Garrett WS, et al. Metagenomic biomarker discovery and explanation. Genome Biol. (2011) 12:R60. doi: 10.1186/gb-2011-12-6-r60

9. Douglas GM, Beiko RG, Langille MGI. Predicting the functional potential of the microbiome from marker genes using PICRUSt. Methods Mol. Biol. (2018) 1849:169-77. doi: 10.1007/978-1-4939-8728-3_11

10. Bianchi E, Wright GJ. Sperm meets egg: the genetics of mammalian fertilization. Annu. Rev. Genet. (2016) 50:93-111. doi: 10.1146/annurev-genet-121415-121834 draft of the manuscript. ZZ made a contribution to the recent revision for this manuscript. All authors approved the final version of the manuscript.

\section{FUNDING}

This study was supported by the National Key Research and Development Program of China (2019YFA0801403), National Nature Science Foundation of China (Nos. 81771660 and 81741017), the Science and Technology Planning Project of Guangdong Province (2017A020214018 and 2017A020214003), Guangdong Natural Science Foundation (2018A030313023, 2018A030313162, 2018A030310162, and 18zxxt56), the Science and Technology Planning Project of Guangzhou City Central Universities (201704020034), 5010 projects at Sun Yat-Sen University (2012006), Science and Technology Planning Project of Guangdong Province (pdjh2020b0010).

\section{SUPPLEMENTARY MATERIAL}

The Supplementary Material for this article can be found online at: https://www.frontiersin.org/articles/10.3389/fimmu. 2021.641281/full\#supplementary-material

11. Walther-António MRS, Chen J, Multinu F, Hokenstad A, Distad TJ, Cheek $\mathrm{EH}$, et al. Potential contribution of the uterine microbiome in the development of endometrial cancer. Genome Med. (2016) 8:122. doi: 10.1186/s13073-016-0368-y

12. Yang S, Gao X, Meng J, Zhang A, Zhou Y, Long M, et al. Metagenomic analysis of bacteria, fungi, bacteriophages, and helminths in the gut of giant pandas. Front. Microbiol. (2018) 9:1717. doi: 10.3389/fmicb.2018.01717

13. Mandal S, Godfrey KM, McDonald D, Treuren WV, Bjornholt JV, Midtvedt $\mathrm{T}$, et al. Fat and vitamin intakes during pregnancy have stronger relations with a pro-inflammatory maternal microbiota than does carbohydrate intake. Microbiome. (2016) 4:55. doi: 10.1186/s40168-016-0200-3

14. Tapiainen T, Paalanne N, Tejesvi MV, Koivusaari P, Korpela K, Pokka T, et al. Maternal influence on the fetal microbiome in a population-based study of the first-pass meconium. Pediatr. Res. (2018) 84:371-9. doi: 10.1038/pr.2018.29

15. Antwis RE, Edwards KL, Unwin B, Walker SL, Shultz S. Rare gut microbiota associated with breeding success, hormone metabolites and ovarian cycle phase in the critically endangered eastern black rhino. Microbiome. (2019) 7:27. doi: 10.1186/s40168-019-0639-0

16. Katagiri S, Shiba T, Tohara H, Yamaguchi K, Hara K, Nakagawa K, et al. Re-initiation of Oral food intake following enteral nutrition alters oral and gut microbiota communities. Front. Cell Infect. Microbiol. (2019) 9:434. doi: $10.3389 /$ fcimb.2019.00434

17. Jedrzejas MJ, Huang WJM. Bacillus species proteins involved in spore formation and degradation: from identification in the genome, to sequence analysis, and determination of function and structure. Crit. Rev. Biochem. Mol. Biol. (2003) 38:173-98. doi: 10.1080/713609234

18. Mongkolthanaruk W. Classification of bacillus beneficial substances related to plants, humans and animals. J. Microbiol. Biotechnol. (2012) 22:1597-604. doi: 10.4014/jmb.1204.04013

19. De la Cruz-Barron M, Cruz-Mendoza A, Navarro-Noya YE, Ruiz-Valdiviezo VM, Ortiz-Gutierrez D, Ramirez-Villanueva DA, et al. The bacterial community structure and dynamics of carbon and nitrogen when maize (Zea mays L.) and its neutral detergent fibre were added to soil from zimbabwe with contrasting management practices. Microb. Ecol. (2017) 73:135-52. doi: $10.1007 / \mathrm{s} 00248-016-0807-8$ 
20. Nagaraj V, Skillman L, Ho G, Li D, Gofton A. Characterisation and comparison of bacterial communities on reverse osmosis membranes of a full-scale desalination plant by bacterial $16 \mathrm{~S}$ rRNA gene metabarcoding. NPJ Biofilms Microbiomes. (2017) 3:13. doi: 10.1038/s41522-017-0021-6

21. Rautava S, Luoto R, Salminen S, Isolauri E. Microbial contact during pregnancy, intestinal colonization and human disease. Nat. Rev. Gastroenterol. Hepatol. (2012) 9:565-76. doi: 10.1038/nrgastro.20 12.144

22. Roberto Romero M, Francesca Gotsch MD, Beth Pineles BS, Juan Pedro Kusanovic MD. Inflammation in pregnancy: its roles in reproductive physiology, obstetrical complications, and fetal injury. Nutr. Rev. (2007) 65:535-43. doi: 10.1301/nr.2007.dec.S194-S202

23. Alijotas-Reig J, Esteve-Valverde E, Ferrer-Oliveras R, Llurba E, Gris JM. Tumor necrosis factor-alpha and pregnancy: focus on biologics. An updated and comprehensive review. Clin. Rev. Allergy Immunol. (2017) 53:40-53. doi: $10.1007 / \mathrm{s} 12016-016-8596-x$
24. Hoya M, Nagamatsu T, Fujii T, Schust DJ, Oda H, Akiba N, et al. Impact of Th1/Th2 cytokine polarity induced by invariant NKT cells on the incidence of pregnancy loss in mice. Am. J. Reprod. Immunol. (2018) 79:e12813. doi: 10.1111/aji.12813

Conflict of Interest: The authors declare that the research was conducted in the absence of any commercial or financial relationships that could be construed as a potential conflict of interest.

Copyright (c) 2021 Bai, Huang, Fu, Zhu, Hu, Zhu, Chen, Zhang, Tan, Zhang and Chen. This is an open-access article distributed under the terms of the Creative Commons Attribution License (CC BY). The use, distribution or reproduction in other forums is permitted, provided the original author(s) and the copyright owner(s) are credited and that the original publication in this journal is cited, in accordance with accepted academic practice. No use, distribution or reproduction is permitted which does not comply with these terms. 